\title{
LAST MINUTE RESULTS FROM CTIO OBSERVATIONS
}

\author{
R. BARBA ${ }^{1, *,+}$ and J. SAHADE ${ }^{1,2, \#,+}$ \\ C.C. 677, Observatorio Astronómico \\ 1900 La Plata, Argentina \\ Instituto Argentino de Radioastronomía \\ C.C. 5, 1894 Villa Elisa, Bs. As., Argentina
}

From June 26 through July 1, 1991 observations were performed at the Cerro Tololo Interamerican Observatory of two peculiar binaries that migth be in different phases of the rapid mass loss stage of evolution, namely, R ARAE and W SERPENTIS.

The material was obtained with the fiber-fed bench-mounted CCD échelle spectrograph at the $1.52 \mathrm{~m}$ telescope and cover the spectral region 5500-7000 $\AA$. A TEK 1024 CCD was used and the spectral resolution was 27,000 .

The assigned observing permitted us to run the observations throughout more than one complete orbital cycle of $\mathrm{R}$ Arae and through a principal minimum of $\mathrm{W}$ Serpentis. Figures 1 and 2 display sets of spectra of $\mathrm{R}$ Arae and W Serpentis that illustrate the rapid changes undergone by the He I 5876 and $\mathrm{H}_{\alpha}$ features. The phases were computed with the expressions,

$$
\operatorname{MinI}=J D \quad 2,447,779.989+4.425 E
$$

(Giménez et al. 1990), for R Arae, and

$$
M \text { inI }=J D 2,426,625.493+14.15486 E+3.140 \times 10^{-6} E^{2}+1.432 \times 10^{-9} E^{3}
$$

(Koch and Guinan 1978), for W Ser.

\section{References}

Giménez, A., González-Riestra, R., Guinan, E.F., Kondo, Y., McCluskey, G., Bradstreet, D.H., McCook, G.P., Dorren, J.D., Johansson, S. and Sahade J. 1990, in IUE Astronomy in the Era of New Space Missions, ESA SP-310, ed. E. Rolfe, p.383.

Koch, R.H. and Guinan E.F. 1978, IBVS $\mathrm{N}^{\circ} 1483$.

* On a Fellowship from CONICET, Argentina

\# Member of the Carrera del Investigador Científico, CONICET, Argentina.

+ Visiting Astronomer, Cerro Tololo Interamerican Observatory, National Optical Astronomy Observatories, opperated by the Association of Universities for Research in Astronomy, Inc., under contract with the National Science Foundation. 


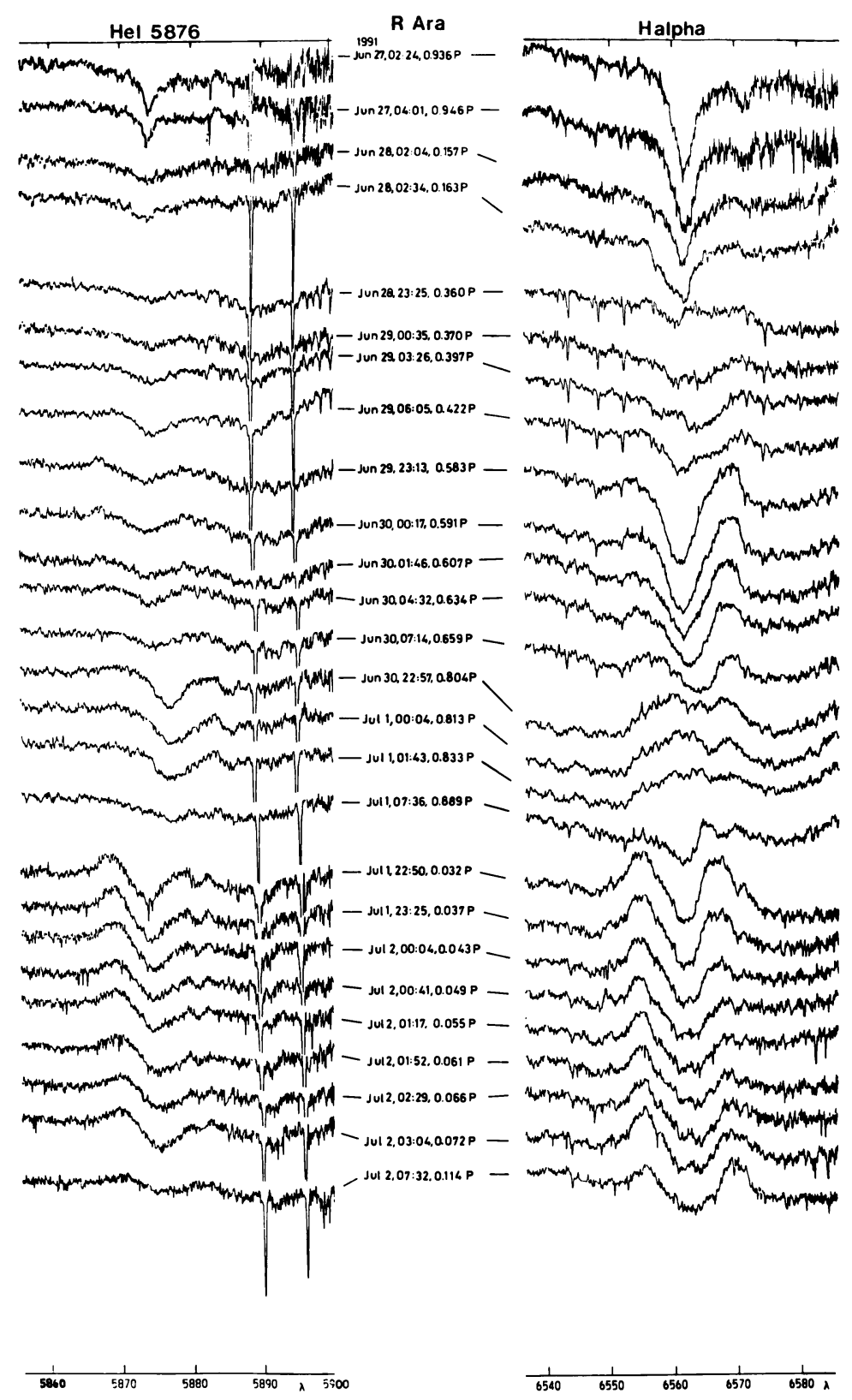

Figure 1. Illustrative set of the spectra of $\mathrm{R}$ Arae taken with the fiber-fed bench-mounted CCD échelle spectrograph at the $1.52 \mathrm{~m}$ telescope at Cerro Tololo on June 26-July1, 1991. The label for each set of profiles gives date, UT of mid-exposure and phase, respectively. 


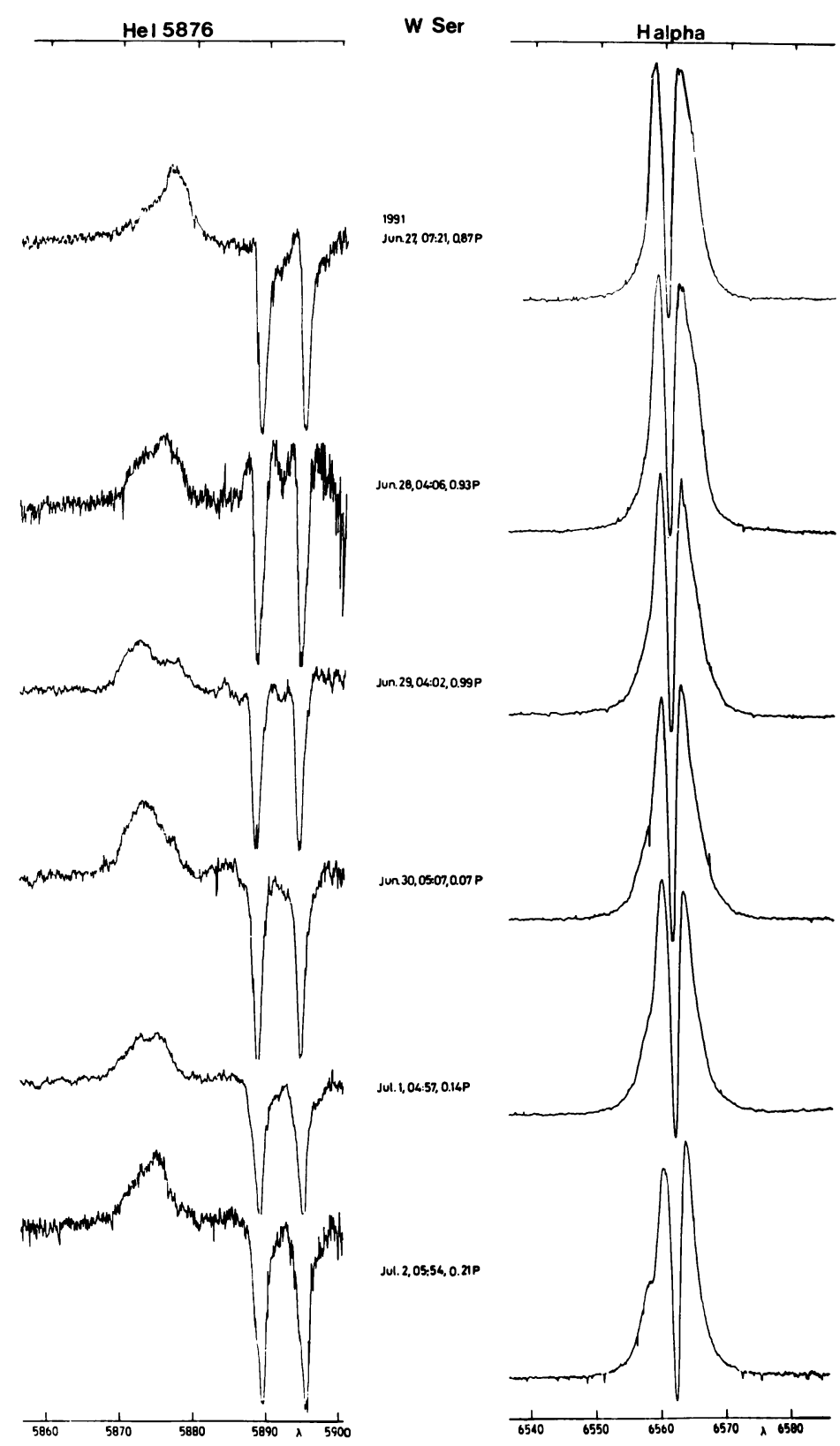

Figure 2. Illustrative set of the spectra of W Serpentis taken with the fiber-fed bench-munted CCD échelle spectrograph at the $1.52 \mathrm{~m}$ telescope at Cerro Tololo on June 26-July1, 1991. The label for each set of profiles gives date, UT of mid-exposure and phase, respectively. 